\title{
Evaluating Glycemic Control in Patients with Type 2 Diabetes Suboptimally Controlled on Basal Insulin: UK ATTAIN Real-World Study
}

\author{
Edward B. Jude · Mark Nixon · Caroline O'Leary · Melissa Myland • \\ Nick Gooch · Alka Shaunik · Elisheva Lew
}

Received: April 29, 2019 / Published online: July 18, 2019

(C) The Author(s) 2019

\begin{abstract}
Introduction: This retrospective, observational cohort study evaluated the effect of therapy intensification on change in glycated hemoglobin (HbA1c) at 6 and 12 months post intensification in patients with type 2 diabetes (T2D) suboptimally controlled on basal insulin (BI) (i.e., HbA1c $\geq 7.5 \%$ [ $\geq 58 \mathrm{mmol} / \mathrm{mol}]$ ).

Methods: Patients with T2D with suboptimal glycemic control using BI were identified from The Health Improvement Network (THIN) database. Patients who underwent therapy intensification (intensifiers) within 12 months of index 1
\end{abstract}

Enhanced Digital Features To view enhanced digital features for this article go to https://doi.org/10.6084/ m9.figshare.8685155.

E. B. Jude $(\varangle)$

Diabetes Centre, Tameside General Hospital,

Ashton-under-Lyne, UK

e-mail: Edward.Jude@tgh.nhs.uk

E. B. Jude

University of Manchester, Manchester, UK

M. Nixon · C. O'Leary · M. Myland

IQVIA, London, UK

N. Gooch

Sanofi, Guildford, UK

A. Shaunik

Sanofi, Bridgewater, NJ, USA

E. Lew

Sanofi, Chilly-Mazarin, France (the date of the first incidence of suboptimally controlled HbA1c) were matched (1:1) to patients who did not intensify therapy (non-intensifiers). Index 2 was the date of therapy intensification for intensifiers, or a pseudo date for non-intensifiers that resulted in the same duration from index 1 to index 2 as their matched intensifier patient. Primary outcomes were HbA1c change and proportion of patients achieving the HbA1c target at 6 and 12 months post index 2 .

Results: A total of 1342 patients $(n=646$ intensifiers; $n=696$ non-intensifiers) were included in the analysis. At post index 2, mean HbA1c change was substantially greater at 6 months for intensifiers than for non-intensifiers $(-0.81 \%$ vs. $-0.35 \%)$, with no additional benefit at 12 months $(-0.81 \%$ vs. $-0.49 \%$, respectively). Compared with non-intensifiers, a greater proportion of intensifiers achieved target HbA1c at 6 months (25.1\% vs. $18.8 \%)$ and at 12 months (33.4\% vs. $28.2 \%$ ).

Conclusions: Many real-world patients with T2D suboptimally controlled with BI do not have their therapy intensified. The results of this study suggest that in this patient population, therapy intensification achieves significant reductions in $\mathrm{HbA1c}$ at 6 months post intensification, with little additional clinical benefit at 12 months. This suggests that, for patients who fail to achieve their glycemic targets at 6 months, since no meaningful additional clinical benefit is observed at 12 months when continuing the same therapy, further 
therapy intensification or change should be promptly considered.

Funding: This study and the Rapid Service Fees were funded by Sanofi.

Trial registration: 17THIN068.

Keywords: Basal insulin; Glycemic control; Therapy intensification; Type 2 diabetes

\section{INTRODUCTION}

According to Quality Outcomes Framework (QOF) of Diabetes UK, an estimated 3.7 million patients in the UK are currently living with diabetes [1], and this number is predicted to increase in the future [2]. These findings will have implications on the burden of diabetes complications (heart disease, stroke, visual impairment, chronic kidney disease, peripheral vascular disease) that can reduce the quality of life and shorten life expectancy [3]. Such complications have been strongly associated with increasing glycated hemoglobin (HbA1c) levels among patients with type 2 diabetes (T2D) [4].

As many as half of those treated for T2D do not achieve the specified targets for HbA1c $[5,6]$ and consequently require therapy intensification [7]. In line with American Diabetes Association (ADA) guidelines [8], the National Institute for Health and Care Excellence (NICE) guidelines for management of T2D in adults recommend that HbA1c levels be measured at 3- to 6-month intervals-as appropriate-following therapy initiation. NICE recommends that patients' HbA1c targets be adjusted according to risk of hypoglycemia, with intensification being considered if the HbA1c level rises above $7.5 \%(58 \mathrm{mmol} / \mathrm{mol})$ [7]. If control is not achieved with sequential dual oral therapy, adding on a third non-insulin therapy (triple therapy) or any insulin-containing therapy combination is recommended [7]. NICE also recommends rescue therapy with insulin or a sulfonylurea at any phase if patients are symptomatically hyperglycemic [7]. For comparison, the ADA HbA1c target is $<7.0 \%(53 \mathrm{mmol} / \mathrm{mol})$ for most nonpregnant adults, with intensification strategies depending on various patient factors. Additionally, if $\mathrm{HbA} 1 \mathrm{c}$ is $>10.0 \%$ or the hyperglycemia is symptomatic, ADA guidelines highlight that insulin can be considered immediately [8].

Despite clear NICE recommendations, clinical control of HbA1c levels in patients with diabetes in the UK often remains suboptimal $(\geq 7.5 \% \quad[\geq 58 \mathrm{mmol} / \mathrm{mol}]), \quad$ and therapeutic inertia may exist for prolonged periods of time [9-12]. Successful glycemic control has been shown to reduce the risk of T2D-related mortality, myocardial infarction, and microvascular complications [13]. However, little research has been undertaken to assist healthcare providers in judging the appropriate timing for therapy intensification in clinical practice.

The aim of this retrospective observational cohort study was to evaluate the effect of therapy intensification (vs. no intensification) on HbA1c levels at 6 and 12 months in patients with T2D suboptimally controlled on basal insulin (BI) and to evaluate the proportion of patients attaining HbA1c targets. The findings will provide information to healthcare providers on the optimal timing for therapy intensification for patients with T2D whose glycemic control remains suboptimal on BI.

\section{METHODS}

\section{Study Design and Data Source}

The Health Improvement Network (THIN) database was used in this retrospective observational cohort analysis. This is a UK primary care database of pseudonymized electronic medical records from 3.1 million actively registered patients in 390 general practitioner practices as of January 2017. THIN was considered to be a suitable data source for this study as T2D is managed in the primary care setting in the UK [7], and the prevalence of T2D in this database is comparable with national estimates for the UK [14]. The study protocol was approved by the THIN Scientific Review Committee under submission 17THIN068. 


\section{Study Cohorts}

Patients aged $\geq 18$ years with T2D and suboptimal glycemic control who had been prescribed BI between 1 January 2005 and 16 May 2017 were identified from the THIN database. The date of the first incidence of suboptimally controlled HbA1c was recorded as index 1 for each patient. For patients who intensified therapy (termed 'intensifiers'), the date of therapy intensification was recorded as index 2 .

A control cohort of patients with suboptimally controlled HbA1c who did not intensify therapy (termed 'non-intensifiers') were matched on a 1:1 basis with intensifiers. Exact matching was performed between the two groups according to: age group at baseline (as per Table 1); sex; baseline HbA1c category (7-7.9, 8-8.9, 9-9.9, 10-10.9, 11-11.9, $\geq 12 \%$ [53-63, 64-74, 75-85, 86-96, 97-107, $\geq 108 \mathrm{mmol} / \mathrm{mol}$, respectively]); number of unique prescribed antihyperglycemia drugs at index $1(1,2$, or $\geq 3)$; and time from first T2D diagnosis to index 1 .

Therapy intensification was defined as the addition of a glucagon-like peptide-1 receptor agonist (GLP-1 RA) or a rapid-acting insulin (RAI) to current treatment during the 12 months following index 1. Patients without evidence of such therapy additions were classified as nonintensifiers. For non-intensifiers, a pseudo date within 12 months of index 1 was assigned as index 2 . This date was chosen so that the duration from index 1 to index 2 was the same as that of the matching intensified patient.

Baseline HbA1c was the HbA1c measurement at index 1 (Fig. 1). Suboptimal HbA1c control was defined as $\mathrm{HbA} 1 \mathrm{c}>7.5 \%$ (> $58 \mathrm{mmol} / \mathrm{mol})$ for patients not at high risk of hypoglycemia and as $\mathrm{HbA} 1 \mathrm{c}>8.0 \% \quad(>64 \mathrm{mmol} / \mathrm{mol})$ for those at high risk of hypoglycemia; HbA1c targets for the groups were $\leq 7.5$ and $\leq 8.0 \%$, respectively. High-risk patients had a history of hypoglycemic coma pre index 1 or they were aged $>65$ years with $\geq 1$ of the following conditions prior to index 1: congestive heart disease, non-hemorrhagic stroke, myocardial infarction/coronary heart disease, peripheral vascular disease, or renal dysfunction.

Patients had $\geq 180$ days of clinical data prior to index 1 (baseline) and $\geq 365$ days of clinical data after index 2 (follow-up). Patients were excluded if the HbA1c level improved to below the target level between index 1 and index 2, if they became pregnant during the study period, or if they had received therapy intensification in the baseline period prior to index 1 .

\section{Outcomes of Interest}

Primary outcomes were: (1) changes in HbA1c at 6 months and 12 months post index 2 , and (2) the proportion of patients achieving target $\mathrm{HbA} 1 \mathrm{c}$ at 6 and 12 months post index 2 .

Other outcomes of interest included comparisons of therapy adherence (proportion of days covered [PDC]) and persistence (time to discontinuation) pre index 1 (baseline) and post index 2 , and assessment of the impact of delay in initiation of therapy intensification.

\section{Statistical Analysis}

The difference in HbA1c change between the two groups post index 2 was calculated using summary statistics and unadjusted/adjusted mixed model repeated measure (MMRM) estimates. Adjusted logistic regression was used to compare the proportion of patients achieving the target HbA1c during the 12 months post index 2. A Cox proportional hazards model was used to describe time to target HbA1c attainment (presented using a Kaplan-Meier curve). As a Bonferroni adjustment was applied to all $P$ values based on 100 tests conducted, $P<0.0005$ was considered to be significant.

$\mathrm{BI}$ adherence at baseline was defined as the PDC in the 180 days prior to index 1 , calculated as the days supplied divided by the total number of follow-up days, excluding any overlapping days supplied. Days supplied was taken either directly from patients' medical records or calculated based on quantity supplied and daily dose. The formula defined by Basak et al. [15] was used to account for overlapping combinations of prescriptions. Adherence to intensification therapy post index 2 was measured by the PDC for the intensification therapy during a 365-day follow-up period and included the use of any drug qualifying as therapy intensification 
Table 1 Baseline demographics and characteristics

\begin{tabular}{|c|c|c|c|c|c|}
\hline \multirow[t]{2}{*}{ Characteristic } & \multirow{2}{*}{$\begin{array}{l}\text { Overall } \\
(n=1342)\end{array}$} & \multicolumn{3}{|c|}{ Intensified therapy } & \multirow{2}{*}{$\begin{array}{l}\text { Not intensified } \\
\text { therapy } \\
(n=696)\end{array}$} \\
\hline & & $\begin{array}{l}\text { GLP-1 RA } \\
(n=55)\end{array}$ & $\begin{array}{l}\text { RAI } \\
(n=591)\end{array}$ & $\begin{array}{l}\text { Total } \\
(n=646)\end{array}$ & \\
\hline Mean age, years (SD) & $58.7(13.1)$ & $55.1(10.1)$ & $58.6(13.1)$ & $58.3(12.9)$ & $59.0(13.3)$ \\
\hline \multicolumn{6}{|l|}{ Age range, years, $n(\%)$} \\
\hline $18-44$ & $198(14.8)$ & $8(14.5)$ & $86(14.6)$ & $94(14.6)$ & $104(14.9)$ \\
\hline $45-54$ & $308(23.0)$ & $18(32.7)$ & $132(22.3)$ & $150(23.2)$ & $158(22.7)$ \\
\hline $55-64$ & $391(29.1)$ & $21(38.2)$ & $169(28.6)$ & $190(29.4)$ & $201(28.9)$ \\
\hline$\geq 65$ & $445(33.2)$ & $8(14.5)$ & $204(34.5)$ & $212(32.8)$ & $233(33.5)$ \\
\hline Male, $n(\%)$ & $718(53.5)$ & $27(49.1)$ & $316(53.5)$ & $343(53.1)$ & $375(53.9)$ \\
\hline Mean HbAlc, \% (SD) & $9.39(1.52)$ & $9.37(1.31)$ & $9.38(1.47)$ & $9.38(1.45)$ & $9.40(1.57)$ \\
\hline \multicolumn{6}{|l|}{ HbAlc category, $n(\%)$} \\
\hline 7.5 to $<9.0 \%(58-<75 \mathrm{mmol} / \mathrm{mol})$ & $631(47.0)$ & $24(43.6)$ & $281(47.5)$ & $305(47.2)$ & $326(46.8)$ \\
\hline 9.0 to $<10.0 \%(75-<86 \mathrm{mmol} / \mathrm{mol})$ & $312(23.2)$ & $15(27.3)$ & $136(23.0)$ & $151(23.4)$ & $161(23.1)$ \\
\hline$\geq 10.0 \%(\geq 86 \mathrm{mmol} / \mathrm{mol})$ & $399(29.7)$ & $16(29.1)$ & $174(29.4)$ & $190(29.4)$ & $209(30.0)$ \\
\hline \multicolumn{6}{|l|}{ Duration of diabetes, $n(\%)$} \\
\hline$<5$ years & $107(8.0)$ & $-^{\mathrm{a}}$ & $51(8.6)$ & $51(7.9)$ & $56(8.0)$ \\
\hline 5 to $<10$ years & $433(32.3)$ & $-^{\mathrm{a}}$ & $200(33.8)$ & $200(31.0)$ & $233(33.5)$ \\
\hline$\geq 10$ years & $780(58.1)$ & $33(60.0)$ & $340(57.5)$ & $373(57.7)$ & $407(58.5)$ \\
\hline \multicolumn{6}{|l|}{ Number of antihyperglycemia drugs, $n$ (\%) } \\
\hline 1 & $589(43.9)$ & $-{ }^{\mathrm{a}}$ & $277(46.9)$ & $277(42.9)$ & $312(44.8)$ \\
\hline 2 & $577(43.0)$ & $29(52.7)$ & $254(43.0)$ & $283(43.8)$ & $294(42.2)$ \\
\hline$\geq 3$ & $150(11.2)$ & $-{ }^{\mathrm{a}}$ & $60(10.2)$ & $60(9.3)$ & $90(12.9)$ \\
\hline Mean weight, kg (SD) & $90.3(21.3)$ & $110.8(25.8)$ & $88.0(20.2)$ & $90.2(21.8)$ & $90.4(20.9)$ \\
\hline Mean BMI, kg/m² (SD) & $32.0(7.3)$ & $39.2(8.6)$ & $31.0(6.7)$ & $31.8(7.3)$ & $32.1(7.4)$ \\
\hline \multicolumn{6}{|l|}{ Comorbidities, $n$ (\%) } \\
\hline Hypertension & $137(10.2)$ & $-{ }^{\mathrm{a}}$ & $63(10.7)$ & $63(9.8)$ & $74(10.6)$ \\
\hline Dyslipidemia & $23(1.7)$ & $-^{\mathrm{a}}$ & $8(1.4)$ & $8(1.2)$ & $15(2.2)$ \\
\hline Obesity (including baseline BMI $>30 \mathrm{~kg} / \mathrm{m}^{2}$ ) & $500(37.3)$ & $40(72.7)$ & $198(33.5)$ & $238(36.8)$ & $262(37.6)$ \\
\hline
\end{tabular}

$B M I$ body mass index, GLP-1 RA glucagon-like peptide-1 receptor agonist, HbAlc glycated hemoglobin, $R A I$ rapid-acting insulin, $S D$ standard deviation

${ }^{a}$ Indicates patient numbers $<6$ for whom data were not provided in order to maintain anonymity

at index 2. The PDC is commonly used to measure insulin adherence in database studies [16]. In about $63 \%$ of patients, the insulin dose was derived from THIN and, in about $37 \%$ of patients, it was imputed based on data available. Adherence post index 2 was not measured for 


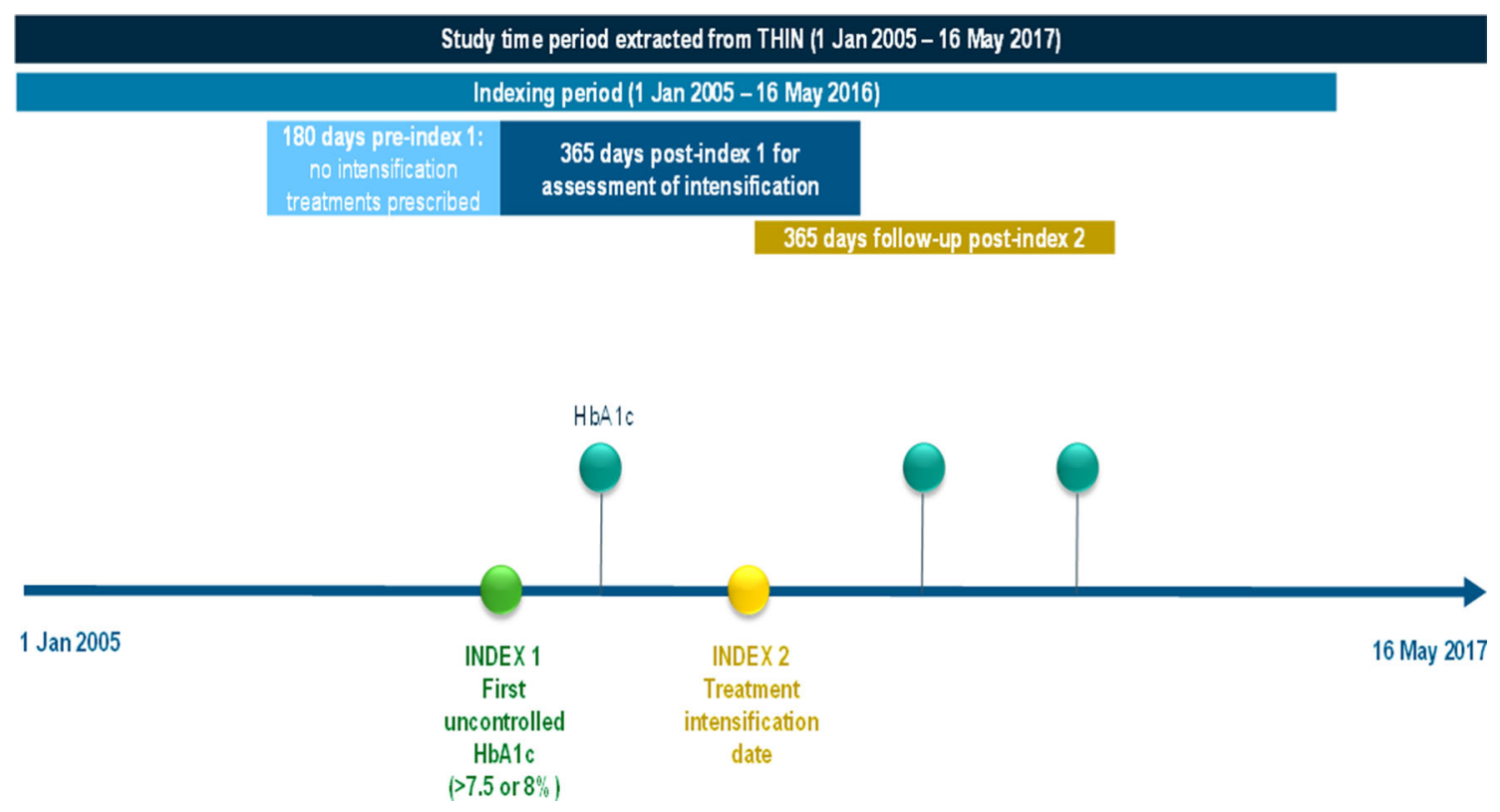

Fig. 1 Study design. HbAlc Glycated hemoglobin, THIN The Health Improvement Network

the non-intensifiers as they did not receive an intensification therapy.

As pharmacy claims data were not available, persistence was defined as a period free of any gap in therapy of $>60$ days post index 2 (gaps of $\leq 60$ days were allowed). Gaps in treatment were calculated based on the time between the calculated end date for a prescription (based on start date and recorded or derived duration) and the start date of the next prescription. If multiple persistent periods were observed post index 2, the first period was used.

Delay in therapy intensification was measured in days from index 1 to index 2 for patients who intensified at any point during the study (i.e., all intensifiers, plus a subset of nonintensifiers who intensified therapy later than 12 months post index 1).

\section{RESULTS}

\section{Baseline Characteristics}

We identified 1342 BI users $(n=646$ intensifiers, $n=696$ non-intensifiers) from the THIN database who met the study inclusion criteria (Fig. 2). Baseline characteristics are shown in
Table 1. Mean baseline HbA1c was similar in the two groups: $9.38 \%$ (standard deviation [SD] $1.45]$ and $9.40 \%$ (SD 1.57), respectively. Overall, $90 \%$ of patients were assigned a target $\mathrm{HbA} 1 \mathrm{c}$ of $7.5 \%$ at baseline, with the reminder being assigned a target HbA1c of $8.0 \%$.

Among the intensifiers, $91.5 \%$ added a RAI and $8.5 \%$ added a GLP-1 RA to their BI therapy. Mean weight, body mass index (BMI), and prevalence of obesity were all higher in the subgroup of intensifiers who added a GLP-1 RA than in the subgroup of non-intensifiers. This observation is consistent with NICE guideline recommendations that adults with $\mathrm{T} 2 \mathrm{D}$ who have a $\mathrm{BMI} \geq 35 \mathrm{~kg} / \mathrm{m}^{2}$ should receive a GLP-1 RA [7].

\section{Glycemic Control}

Improvement in $\mathrm{HbA1c}$ at 6 months was substantially greater for intensifiers (mean [SD] $-0.81 \% \quad[1.7 \%])$ than for non-intensifiers $(-0.35 \%$ [1.7\%]) (Fig. 3a). HbA1c change at 12 months was also greater for intensifiers (mean [SD] $-0.81 \%$ [2.0\%]) versus non-intensifiers $(-0.49 \%[2.1 \%]$ ) (Fig. 3b), but without 


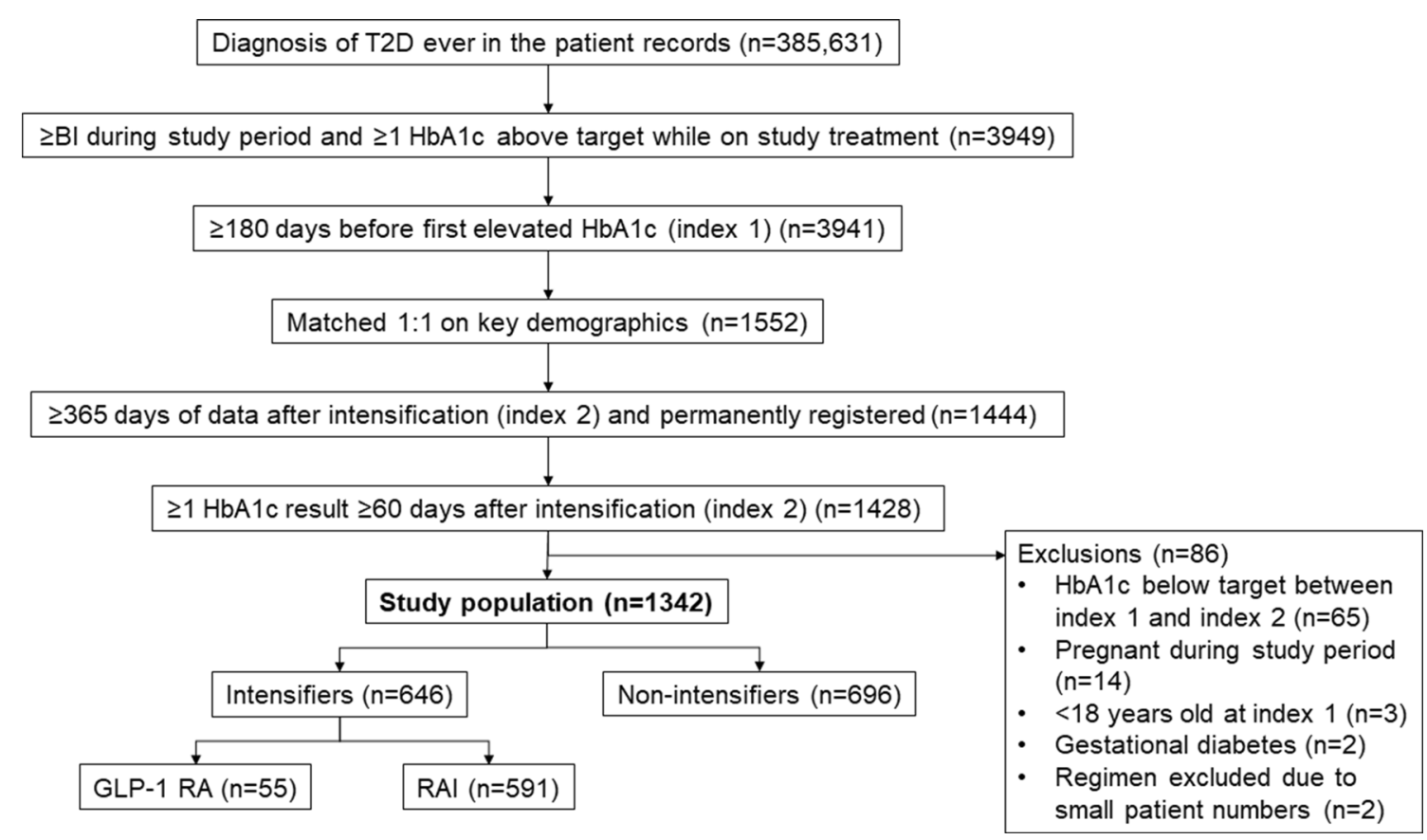

Fig. 2 Patient disposition. $B I$ basal insulin, $G L P-1 R A$ glucagon-like peptide- 1 receptor agonist, $R A I$ rapid-acting insulin, $T 2 D$ type 2 diabetes

additional gain/improvement as compared with the 6-month assessment.

The unadjusted MMRM estimate for therapy difference for HbA1c change was - 0.37\% (95\% confidence interval [CI] $-0.55 \%,-0.18 \%$; $P=0.0002)$ at 6 months, and $-0.33 \%(95 \% \mathrm{CI}$ $-0.52 \%,-0.14 \% ; P=0.0007)$ at 12 months. The adjusted MMRM estimate for therapy difference for HbA1c change was - 0.41\% (95\% CI $-0.64 \%,-0.17 \% ; P=0.0006)$ at 6 months and $-0.32 \% \quad(95 \% \quad$ CI $\quad-0.55 \%, \quad-0.08 \%$; $P=0.0077)$ at 12 months.

A higher proportion of intensifiers versus non-intensifiers achieved target $\mathrm{HbA1c}$ at 6 months $(25.1 \%$ vs. $18.8 \%$, respectively; $P<0.0001$ by logistic regression) and at 12 months $(33.4 \%$ vs. $28.2 \%$, respectively; $P<0.0001$ by logistic regression). At 12 months, the target HbA1c was achieved by a higher proportion of intensifiers receiving GLP-1 RA versus those receiving RAI $(45.5 \%$ vs. $32.3 \%$, respectively), although the proportions were low.

A Kaplan-Meier analysis showed a numerically greater probability of HbA1c target achievement for intensifiers at 6 months that was maintained at 12 months (Fig. 4). The adjusted hazard ratios for target achievement was $0.81(95 \%$ CI $0.61,1.06 ; P=0.13)$ at 6 months and 0.85 (95\% CI $0.67,1.09 ; P=0.20)$ at 12 months.

The mean time to therapy intensification was 9.7 months for patients who intensified with a RAI and 23.2 months for those who intensified with a GLP-1 RA (Table 2).

\section{Medication Adherence and Persistence}

Baseline adherence to BI therapy (the PDC in the 180 days prior to index 1) was $73 \%$ (SD $23.9 \%$ ). Adherence to therapy was comparable between intensifiers (71\% [SD 24.2\%]) and non-intensifiers (76\% [SD 23.5\%]; $P<0.0001$ ) at baseline. Patients who intensified therapy with a GLP-1 RA had the greatest adherence to therapy post index 2 (81\%; SD 20.7\%), whereas those who intensified with a RAI had substantially lower adherence (61\%; SD 20.4\%). Mean persistence with intensification therapy in the intensifier group was 595.0 days (SD 792.8). 


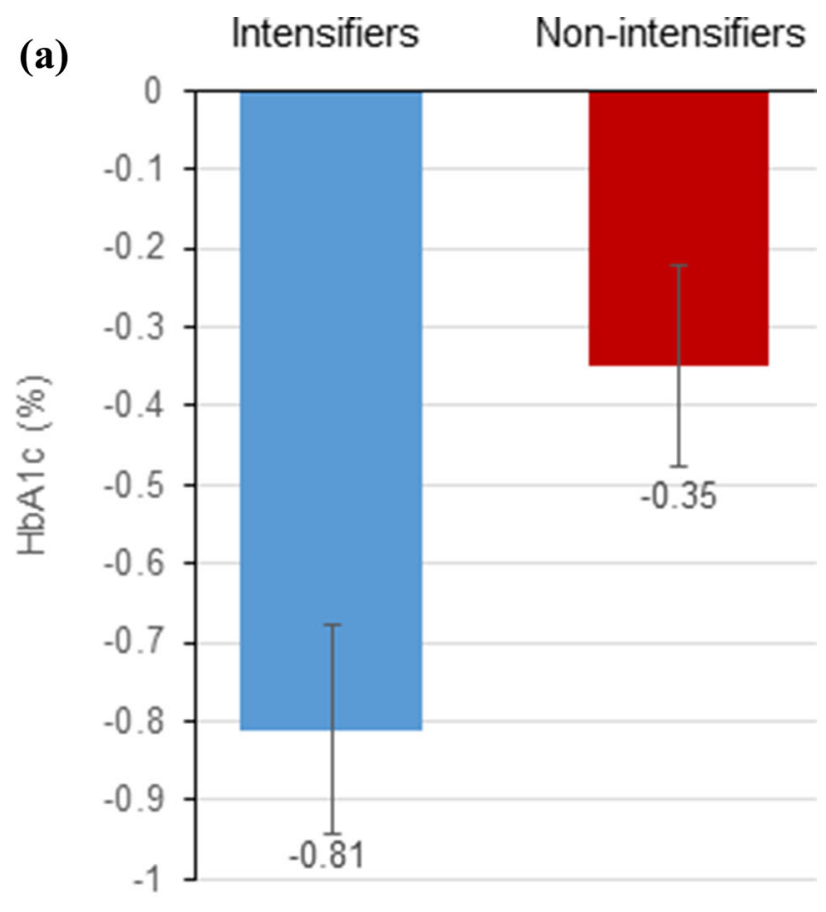

(b)

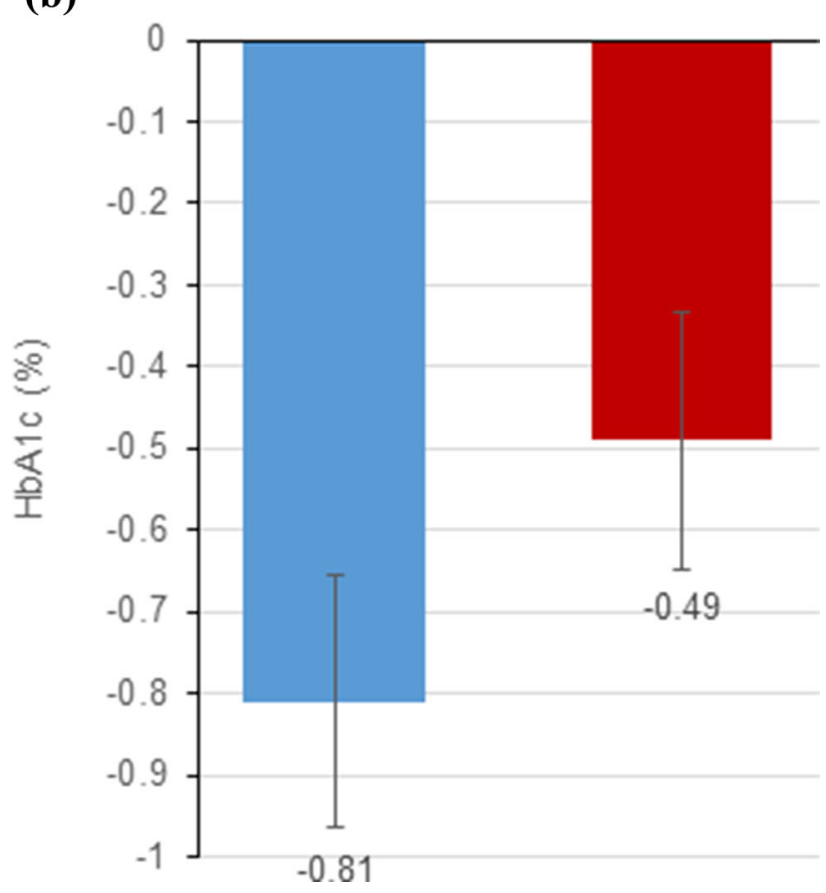

Fig. 3 Change in HbA1c from baseline during the follow-up for intensifiers and non-intensifiers. a At 6 months, $\mathbf{b}$ at 12 months 


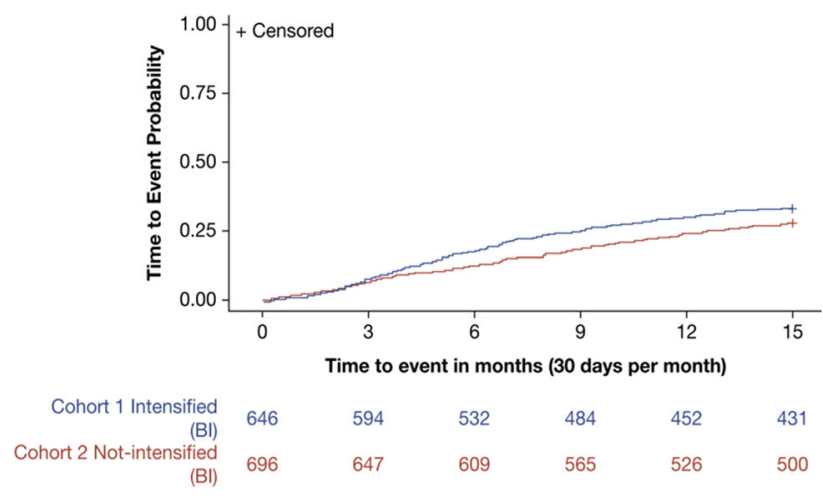

Fig. 4 Kaplan-Meier curve for HbAlc target attainment within 12 months of index 2

Table 2 Duration of delay in therapy intensification

\begin{tabular}{llll}
\hline Characteristic & \multicolumn{2}{l}{ Intensified therapy } \\
\cline { 2 - 3 } & GLP-1 & RAI & Total \\
& RA & $(n=529)$ & intensified \\
& $(n=77)$ & & $(n=606)$
\end{tabular}

Delay in intensification, months

\begin{tabular}{llll} 
Mean & 23.2 & 9.7 & 11.4 \\
SD & 24.4 & 17.2 & 18.8 \\
Median & 10 & 3 & 3 \\
Lower & $4-41$ & $1-10$ & $1-11$ \\
$\begin{array}{l}\text { quartile-upper } \\
\text { quartile }\end{array}$ & & & \\
\hline
\end{tabular}

\section{Weight}

Mean weight at the latest value during the 12 months after index 2 was $107.7 \mathrm{~kg}$ (SD 21.6) and $89.2 \mathrm{~kg}$ (SD 20.6) for those who intensified to GLP-1 RA and RAI, respectively, and $91.8 \mathrm{~kg}$ (SD 21.2) for those who did not intensify, resulting in mean changes of $-3.1,+1.2$, and $+1.4 \mathrm{~kg}$, respectively.

\section{DISCUSSION}

In our study population, patients with T2D suboptimally controlled on BI and who intensified therapy had greater reductions in HbA1c than those who did not receive intensified therapy (non-intensifiers). Most of this improvement occurred within the first
6 months post intensification. Thus, injectable therapy intensification resulted in clinically meaningful improvements in glycemic control. However, a relatively high proportion of both subgroups of the overall study population $(67 \%$ of BI intensifiers and $72 \%$ of non-intensifiers) did not achieve the target HbA1c after 12 months. This observation, coupled with the high mean baseline HbA1c values and the $>6$-month delay in therapy intensification for many after the first detection of suboptimally controlled HbA1c, suggests that there is still a gap between guideline recommendations [7, 8] and real-world practice.

These findings are consistent with those reported in other studies conducted in both the USA and Europe. In a study of a large primary care-based population $(n \approx 12,000)$ in the UK, only $31 \%$ of eligible patients with T2D and HbA1c suboptimally controlled with BI (HbA1c $\geq 7.5 \% \quad[\geq 58 \mathrm{mmol} / \mathrm{mol}])$ underwent therapy intensification during the 9-year study period, with a median time to intensification of 3.7 years [10].

Similar findings were reported by an earlier retrospective analysis of the THIN database that included both patients with controlled and suboptimally controlled T2D who (1) were current BI users and (2) who received a prescription for BI during the period January to June 2006 [11]. During a mean follow-up of 2.9 years, only $19.3 \%$ of patients intensified therapy, whereas $59.8 \%$ remained on their initial BI regimen during the entire follow-up period, despite evidence of poor glycemic control (mean HbA1c $8.4 \%[69 \mathrm{mmol} / \mathrm{mol}]$ at baseline and $8.1 \%$ 
[65 mmol/mol] at the end of follow-up). Patients who underwent intensification had a higher mean HbA1c at baseline $(9.2 \%$ [77 mmol/mol]) but had an HbA1c decrease of $0.6 \%$ from baseline to end of follow-up.

In the present study, early reductions in HbA1c were noted in the non-intensifier cohort that were similar to those seen in the intensifier cohort, but only for the first 3 months. This may have been due to dose adjustments and insulin titration, but data on these treatments were not captured in the current study. Importantly, the intensifier cohort continued to show constant and sustained improvement for the period of observation until 12 months. This HbA1c change and target attainment represent a clinically relevant advantage for patients who intensify therapy when contextualized with the results of the UK Prospective Diabetes Study (UKPDS) study [4], which demonstrated that each $1 \%$ reduction in HbA1c corresponded with a $21 \%$ risk reduction in diabetes-related deaths, a $14 \%$ risk reduction for myocardial infarctions, and a $37 \%$ risk reduction for microvascular complications [4]. More recently, a modeling study estimated 10-year cumulative risk reductions of 5 and $9 \%$ for myocardial infarction, 6 and $13 \%$ for stroke, 14 and $27 \%$ for diabetesrelated ulcer, 22 and 39\% for amputation, and 14 and $25 \%$ for single-eye blindness when $\mathrm{HbA} 1 \mathrm{c}$ is at $9 \%(75 \mathrm{mmol} / \mathrm{mol})$ and $8 \%$ $(64 \mathrm{mmol} / \mathrm{mol})$, respectively, compared with when $\mathrm{HbA} 1 \mathrm{c}$ is at $10 \%(86 \mathrm{mmol} / \mathrm{mol})$ [17]. Given that the baseline HbA1c in the current study was approximately $9.4 \%(79 \mathrm{mmol} / \mathrm{mol})$ and the follow-up HbA1c among intensifiers was approximately $8.6 \%(70 \mathrm{mmol} / \mathrm{mol})$, this result represents clinically meaningful risk reductions.

The Kaplan-Meier analysis indicates that intensifiers had a numerically higher probability of achieving target HbA1c over time, but that a substantial proportion of the population (67\% of BI intensifiers and $72 \%$ of non-intensifiers) had not achieved the target at 12 months post intensification. Even with optimal adherence, previous studies have indicated that approximately $40 \%$ of patients do not achieve their HbA1c targets with BI $[18,19]$. However, these studies occurred in a clinical trial setting and thus differed in terms of measurement frequency and the selected population. Nevertheless, it is clear that therapy intensification with $\mathrm{BI}$ is an inadequate strategy for many patients.

Mean adherence to therapy was somewhat higher at baseline (PDC 73\% overall) than post intensification (PDC 63\% among intensifiers), at which time it was similar to that reported using US claims data, in which insulin users had a medication possession ratio of 0.63 [20]. While there are many methods to measure adherence, which vary from study to study, there is a lack of consensus on the best measure of adherence [21]. Despite this, previous analyses have consistently shown that improved adherence is associated with decreased costs and resource use [21]. A focused literature review described a wide range of adherence rates in the UK ranging from around $43 \%$ to up to $86 \%$, but noted the difficulties in the objective assessment of adherence [22]. In our study, despite appropriate adherence and persistence with therapies, low proportions of patients achieved the target HbA1c within 6 or 12 months of therapy intensification. Thus, realistically, additional clinically relevant reductions in HbA1c are unlikely to be delivered through further improvement in adherence to therapy, as this is already approaching the upper level of what has been previously reported in the literature.

The persistence with therapy reported in this study is of notably longer duration than persistence reported in the literature. A review of a retrospective database study in the USA [23] found that patients adding a RAI to their current therapy remained persistent for only 274 days, approximately half the time observed in the present analysis. Additionally, in a further study in the USA, for those using a GLP-1 $\mathrm{RA}$ and $\mathrm{BI}$, median persistence was 133 days [24], which is less than a quarter of the persistence with therapy observed in our study. However, comparisons between European and American persistence data are complicated by access issues around private health insurance and co-payments, differences in electronic health record data capture, and routine health care practices (among other factors).

There are some key limitations to this study. Differences in disease severity may affect 
comparisons between intensifiers and non-intensifiers, although attempts were made to control for such known confounding variables through matching of patients according to baseline characteristics. Diet, exercise, and lifestyle modifications as recommended to patients could not be taken into account and may have been different between groups. Adherence data were based on prescriptions issued; however, it is not known whether these prescriptions were filled or used as directed. Changes in dose (not analyzed in the study) may have impacted estimations of days' supplied and thus led to over- or underestimations of adherence. Furthermore, adherence was calculated using PDC, which is commonly used in retrospective studies; it is more conservative than other methods such as the medication possession ratio [25]. Changes in BI dose due to titration or changes in oral anti-diabetes drug background therapy were not captured and may have influenced HbA1c levels. Occult episodes of hypoglycemia, not captured in THIN, may have altered patients' behavior and affected glycemic control and target attainment. While individualized glycemic goals were set for patients who were assessed to be at high and low risk for hypoglycemia, it is possible that these goals did not reflect true individualized treatment goals in some cases. Lastly, the clinical benefits and cost implications of the differences in HbA1c goal achievement between intensifiers and non-intensifiers are outside the scope of the current study.

\section{CONCLUSIONS}

Therapy intensification in real-world patients with suboptimally controlled T2D while on BI therapy is associated with $\mathrm{HbA1c}$ improvements and an increased likelihood of achieving HbA1c targets. However, many patients do not have their therapy intensified, although this is recommended in the guidelines, or intensification is delayed. In this study, reductions in HbA1c were evident at 6 months following therapy intensification, although with little additional benefit being seen at 12 months. As most of the HbA1c reduction occurred during the 6 months post intensification, this study demonstrates that improvements in glycemic control are achieved rapidly in this population. These results suggest that patients who fail to achieve their glycemic targets at 6 months are unlikely to benefit from continuing on the current therapy and that therapy intensification or change should be promptly considered.

\section{ACKNOWLEDGEMENTS}

Funding. This study and the Rapid Service Fees were funded by Sanofi. All authors had access to all of the data report and output in this study and take complete responsibility for the integrity of the data and accuracy of the data analysis.

Medical Writing, Editorial, and Other Assistance. The authors received writing/editorial support in the preparation of this article provided by Yunyu Huang, PhD, of Excerpta Medica, funded by Sanofi.

Authorship. All authors participated in the study design. Mark Nixon, Caroline O'Leary, and Melissa Myland acquired and analyzed the data. All authors interpreted the data, contributed to the drafting, critical review and revision of the manuscript. All named authors meet the International Committee of Medical Journal Editors (ICMJE) criteria for authorship for this article, take responsibility for the integrity of the work as a whole, and have given their approval for this version to be published.

Disclosures. Edward B. Jude has received advisory board honoraria and grant/research support from Sanofi and has received speaker honoraria from Novo Nordisk, Takeda, Bayer AG, Eli Lilly, and Boehringer Ingelheim. Mark Nixon is an employee of IQVIA, which was contracted by Sanofi to conduct the analysis. Caroline O'Leary is an employee of IQVIA, which was contracted by Sanofi to conduct the analysis. Melissa Myland is an employee of IQVIA, which was contracted by Sanofi to conduct the analysis. Nick Gooch is an employee of 
Sanofi. Alka Shaunik is an employee of Sanofi. Elisheva Lew is an employee of Sanofi.

Compliance with Ethics Guidelines. The study protocol was approved by the THIN Scientific Review Committee under submission 17 THIN068.

Data Availability. The datasets generated during and/or analyzed during the current study are not publicly available due to patient privacy but are available from the corresponding author on reasonable request. THIN is a registered trademark of Cegedim SA in the UK and other countries. Reference made to the THIN database is intended to be descriptive of the data asset licensed by IQVIA. This work uses de-identified data provided by patients as a part of their routine primary care.

Open Access. This article is distributed under the terms of the Creative Commons Attribution-NonCommercial 4.0 International License (http://creativecommons.org/licenses/ by-nc/4.0/), which permits any noncommercial use, distribution, and reproduction in any medium, provided you give appropriate credit to the original author(s) and the source, provide a link to the Creative Commons license, and indicate if changes were made.

\section{REFERENCES}

1. Diabetes UK. Diabetes prevalence. https://www. diabetes.org.uk/professionals/position-statementsreports/statistics/diabetes-prevalence-2017 (2017). Accessed 18 Mar 2019.

2. International Diabetes Foundation. Diabetes atlas http://diabetesatlas.org/IDF_Diabetes_Atlas_8e_int eractive_EN/ (2017). Accessed 18 Mar 2019.

3. Wright KA, Kontopantelus E, Emsley R, et al. Life expectancy and cause-specific mortality in type 2 diabetes: a population-based cohort study quantifying relationships in ethnic subgroup. Diabetes Care. 2017;40:338-45.

4. Stratton IM, Adler AI, Neil HA, et al. Association of glycaemia with macrovascular and microvascular complications of type 2 diabetes (UKPDS 35): prospective observational study. BMJ. 2000;321: 405-12.

5. Jang HC, Guler S, Shestakova M, PRESENT Study Group. When glycaemic targets can no longer be achieved with basal insulin in type 2 diabetes, can simple intensification with a modern premixed insulin help? Results from a subanalysis of the PRESENT study. Int J Clin Pract. 2008;62:1013-8.

6. Avramopoulos I, Moulis A, Nikas N. Glycaemic control, treatment satisfaction and quality of life in type 2 diabetes patients in Greece: the PANORAMA study Greek results. World J Diabetes. 2015;6:208-16.

7. National Institute of Clinical and Care Excellence. Type 2 diabetes in adults: management. https:// www.nice.org.uk/guidance/ng28/chapter/1-recomm endations (2017). Accessed 18 Mar 2019.

8. American Diabetes Association. Standards of medical care in diabetes-2019. Diabetes Care. 2019;42[Suppl. 1]:S1-183.

9. UKPDS Group. Intensive blood-glucose control with sulphonylureas or insulin compared with conventional treatment and risk of complications in patients with type 2 diabetes (UKPDS 33). Lancet. 1998;352:837-54.

10. Khunti K, Nikolajsen A, Thorsted BL, Andersen M, Davies MJ, Paul SK. Clinical inertia with regards to intensifying therapy in people treated with basal insulin. Diabetes Obes Metab. 2016;18:401-9.

11. Blak B, Smith HT, Hards M, Curtis BH, Ivanyi T. Optimization of insulin therapy in patients with type 2 diabetes mellitus: beyond basal insulin. Diabetes Med. 2012;29:13-20.

12. Khunti K, Wolden ML, Thorsted BL, Andersen M, Davies MJ. Clinical inertia in people with type 2 diabetes: a retrospective cohort study of more than 80,000 people. Diabetes Care. 2013;36:3411-7.

13. Moghissi ES, Korytkowski MT, DiNardo M, et al. American Association of Clinical Endocrinologists and American Diabetes Association consensus statement on inpatient glycemic control. Diabetes Care. 2009;32:1119-31.

14. Blak BT, Thompson M, Dattani H, Bourke A. Generalisability of The Health Improvement Network (THIN) database: demographics, chronic disease prevalence and mortality rates. Inform Prim Care. 2011;19:251-5.

15. Basak R, McCaffrey DJ III, Bentley JP, Przybyla SM, West-Strum D, Banahan BF. Adherence to multiple medications prescribed for a chronic disease: a 
methodological investigation. J Manag Care Spec Pharm. 2014;20:815-23.

16. Stolpe S, Kroes MA, Webb N, Wisniewski T. A systematic review of insulin adherence measures in patients with diabetes. J Manag Care Spec Pharm. 2016;22:1224-46.

17. Mostafa SA, Coleman RL, Agbaje OF, Gray AM, Holman RR, Bethel MA. Modelling incremental benefits on complications rates when targeting lower HbA1c levels in people with Type 2 diabetes and cardiovascular disease. Diabetes Med. 2018;35:72-7.

18. Riddle MC, Rosenstock J, Gerich J, Insulin Glargine 4002 Study Investigators. The treat-to-target trial: randomized addition of glargine or human $\mathrm{NPH}$ insulin to oral therapy of type 2 diabetic patients. Diabetes Care. 2003;26:3080-6.

19. Holman RR, Farmer AJ, Davies MJ, et al. Three-year efficacy of complex insulin regimens in type 2 diabetes. N Engl J Med. 2009;361:1736-47.

20. Chandran A, Bonafede MK, Nigam S, Saltiel-Berzin R, Hirsch LJ, Lahue BJ. Adherence to insulin pen therapy is associated with reduction in healthcare costs among patients with type 2 diabetes mellitus. Am Health Drug Benefits. 2015;8:148-58.
21. Kennedy-Martin T, Boye KS, Peng X. Cost of medication adherence and persistence in type 2 diabetes mellitus: a literature review. Patient Prefer Adher. 2017;11:1103-17.

22. Davies MJ, Gagliardino JJ, Gray LJ, Khunti K, Mohan V, Hughes R. Real-world factors affecting adherence to insulin therapy in patients with type 1 or type 2 diabetes mellitus: a systematic review. Diabet Med. 2013;30:512-24.

23. Miao R, Wei W, Baser O, Xie L. Real world outcomes of adding rapid-acting insulin versus switching to analog premix insulin among US patients with type 2 diabetes treated with insulin glargine. Patient Prefer Adher. 2013;7:951-60.

24. Lin J, Lingohr-Smith M, Fan T. Real-world medication persistence and outcomes associated with basal insulin and glucagon-like peptide 1 receptor agonist free-dose combination therapy in patients with type 2 diabetes in the US. Clinicoecon Outcomes Res. 2016;9:19-29.

25. Raebel MA, Schmittdiel J, Karter AJ, Konieczny JL, Steiner JF. Standardizing terminology and definitions of medication adherence and persistence in research employing electronic databases. Med Care. 2013;51(8 Suppl 3):S11-21. 